\title{
8 \\ The Founder and Allee Effects in the Patch Occupancy Metapopulation Model
}

\author{
Rampal S. Etienne and Lia Hemerik
}

\begin{abstract}
The problem of ever-increasing habitat fragmentation due to human land use calls for a theoretical framework to study the potential dangers and to find ways of combating these dangers. The metapopulation approach, with the Levins model as its patriarch, provides such a framework. A metapopulation is a collection of populations that live in habitat fragments (called patches). These populations can become extinct, but new populations can be established by dispersing individuals from extant populations. If these colonizations can balance these extinctions, metapopulation persistence is possible. In theoretical literature surprisingly little attention has been paid to the colonization term in the Levins model and its extensions. Specifically, the Allee effect (i.e. reduced probability of colonization due to, e.g., reduced probability of finding a mate, or reduced defence against predators) may play a major role although it has not received appropriate attention. In this paper, we study the colonization term in the Levins model and conclude that it describes the founder effect (i.e. stochastic fluctuations in births and deaths of an establishing population causing colonization to fail). We then incorporate the Allee effect in the colonization term and conclude that previous attempts to do so were erroneous because they ignored some difficulties in the model formulation and interpretation. We devise a phenomenological model for the Allee effect that is consistent in both discrete and continuous time. Although the model with Allee effect shows a fold bifurcation in its deterministic formulation (both in discrete and continuous time), suggesting the possibility of sudden metapopulation extinction when the bifurcation parameter is only changed slightly, the model in its stochastic formulation does not fully support this: the expected occupancy and the expected metapopulation extinction time decrease gradually when the number of patches is moderate.
\end{abstract}

\subsection{INTRODUCTION}

As the human population grows, both in number and in its use of natural resources, natural habitat for many organisms is disappearing and becoming fragmented. Not only (rail) roads, but also agricultural, residential and industrial areas fragment previously connected (or even continuous) habitat. Common sense tells us that the answer to habitat fragmentation is 
defragmentation and hence much effort is put into building corridors, of which fauna crossings are just one example. Corridors are conduits connecting two pieces of habitat through an environment of hostile non-habitat. As such, the use of corridors need not be restricted to the animal kingdom; plants can also use them as stepping-stones for their seeds, enabling them to colonize distant habitat. Although corridors may not only act as conduits but also as habitat, filters or even as barriers (Hess and Fischer, 2001), in most cases they are constructed primarily for their conduit function.

Habitat fragmentation and subsequent attempts of habitat defragmentation affect extinction of local populations as well as recolonization of empty but suitable habitat. Because extinction and colonization are the two core ingredients of metapopulation theory, metapopulation theory seems well suited for predicting the consequences of habitat (de)fragmentation. A metapopulation is defined as a population consisting of several more or less loosely connected local populations with colonization and extinction of these local populations analogous to births and deaths of individuals in a population. The father of all metapopulation models is undoubtedly the Levins (1969, 1970) patch occupancy model in which the habitat consists of many distinct patches, which can be either empty or occupied by a population of the species under consideration. An occupied patch can become empty by extinction of the local population and an empty patch can become occupied after colonization by dispersers from extant populations. Because the risk of extinction is spread (Den Boer, 1968), the metapopulation can persist much longer than a local population, if recolonization occurs frequently.

The Levins model has been extended in many ways to study the effects of the extension on metapopulation dynamics. These extensions include the rescue effect (Hanski, 1983; Hanski et al., 1996; Gyllenberg and Hanski, 1997; Etienne, 2000, 2002), the anti-rescue effect (Harding and McNamara, 2002), the patch preference effect (Ray et al., 1991; Etienne, 2000), multiple species interactions (Levins and Culver, 1971; Slatkin, 1974; Sabelis et al., 1991; Nee and May, 1992; Hess, 1994, 1996; Nee et al., 1997; Taneyhill, 2000; Gog et al., 2002; Nagelkerke and Menken, 2002), succession (Amarasekare and Possingham, 2001), heterogeneous habitat (Holt, 1997), the quality of the matrix habitat (Vandermeer and Carvajal, 2001), spatial structure (Hanski and Ovaskainen, 2000; Ovaskainen and Hanski, 2001), and local population dynamics and dynamics of patch formation and destruction (Hastings, 1991; Gyllenberg and Hanski, 1992; Hanski and Zhang, 1993, Hanski and Gyllenberg, 1993; Hastings, 1995; Gyllenberg and Hanski, 1997; Gyllenberg et al., 1997; Keymer et al., 2000). These models are all deterministic. Stochastic versions of the Levins model have also been developed and explored (Day and Possingham, 1995; Gyllenberg and Silvestrov, 1994; Frank and Wissel, 1998; Lande et al., 1998; Ovaskainen, 2001, Etienne and Heesterbeek, 2001; Etienne, 2002; Etienne and Nagelkerke, 2002; Alonso and McKane, 2002). 
In all of these models, little change has been made with respect to the colonization process in the Levins model. That is, the colonization rate or the colonization probability (per unit time) has been taken proportional to the number of, on the one hand, empty patches and, on the other hand, the number of dispersers or, when dispersal is not explicitly modelled, the number of occupied patches (which produce dispersers). Yet, colonization of a patch, i.e. the establishment of a new population, may be hampered by the Allee effect (Wissel, 1994), a reduced or even negative growth rate at small population sizes (Allee, 1931), which may affect this proportionality substantially. The Allee effect can be caused by difficulties in, for example, mate finding, food exploitation (e.g. host resistance can only be overcome by sufficient numbers of consumers) and predator avoidance or defence (Allee, 1931; Courchamp et al., 1999; Stephens and Sutherland, 1999; Stephens et al., 1999; McCarthy, 1997) when population size is small. Colonization may also be hampered by sheer stochasticity: even if the growth rate is positive, stochastic fluctuations in birth and death rates may prevent colonization (Goel and Richter-Dyn, 1974). We will call this the founder effect (not to be confused with Mayr's founder effect in evolutionary biology, although the two concepts are related as they both stress the stochastic nature of colonization), and we will show that it is contained in the Levins model, in contrast to a result obtained by Etienne et al. (2002a). Sometimes, the founder effect is also considered a type of Allee effect (Lande, 1998; Keitt et al., 2001; Harding and McNamara, 2002), although not unanimously (Stephens et al., 1999), so we prefer to use separate terms for the two effects.

The Allee effect has previously been brought into connection with metapopulations although the subject was often only briefly touched upon (Courchamp et al., 1999; Reed, 1999; Stephens et al., 1999; Stephens and Sutherland, 1999; Cronin and Strong, 1999; Keitt et al., 2001), or the metapopulation consisted of only two patches (Gyllenberg et al., 1996; Amarasekare, 1998a; Gyllenberg et al., 1999), or the study involved simulations rather than an analytical description and treatment of a dynamical model (Berec et al., 2001; Brassil, 2001; Etienne et al., 2002b). Amarasekare (1998b) did present and analyze a Levins-type model with Allee effect, but this Allee effect was active at the metapopulation level. That is, analogous to the Allee effect at the population level that reduces the growth rate of the population, Amarasekare (1998b) assumed that the Allee effect at the metapopulation level reduces the growth rate of the metapopulation. As a possible cause of this Allee effect at the metapopulation level, Amarasekare (1998b) mentioned the Allee effect at the population level, but she did not model this explicitly. Ovaskainen and Hanski (2001) also considered the Allee effect at the metapopulation level, possibly caused by an Allee effect at the population level which they modelled as in Hanski's (1994) incidence function model. However, they did not study this in great detail, as exact (analytical) solutions seemed impossible in the spatial context they focussed on (but see 
Ovaskainen et al., 2002). The structured metapopulation model of Gyllenberg et al. (1997) and the model of Gotelli and Kelley (1993) allow for incorporation of the Allee effect, but this has not actually been carried out, except for Harding and McNamara (2002) who studied the Allee effect explicitly in the Gotelli and Kelley (1993) model although they modelled this incorrectly, as we will show below.

Thus, the impact of the Allee effect at the population level on metapopulation dynamics has, to our knowledge, never (or at least hardly) been studied analytically in the Levins framework. One reason may be that it seems very simple to perform and the result seems self-evident: a threshold effect. However, this also applies to many models which have been explored analytically, for example, the Levins model with rescue effect (Hanski, 1983; Hanski et al., 1996; Gyllenberg and Hanski, 1997; Etienne, 2000, 2002; Harding and McNamara, 2002), an effect referring to the ability of immigrants to rescue a population from extinction (Brown and Kodric-Brown, 1977). In these cases, more insight was still gained from a mathematical analysis of the model. Moreover, when a model is extended further, results may no longer be self-evident, because the model has become too complicated. How, for example, will the Allee effect influence metapopulation dynamics when modelled in conjunction with interspecific competition or predator-prey interactions (as in the references mentioned above)? It is important to have a thorough understanding of the impact of the Allee effect when the model is still relatively easily tractable in order to properly evaluate the outcomes in more complicated models. Therefore, we will present a Levins-type model with a population-level Allee effect.

We will start with a review of the Levins model and the more general patch occupancy model, of which it is a special case. We will present deterministic and stochastic formulations of this patch occupancy model, both in discrete and continuous time. The occupancy model enables incorporation of the founder effect and the Allee effect. We will show that this is not straightforward by pointing out where previous attempts (notably Etienne et al., 2002a; Harding and McNamara, 2002) have failed. We will study the dynamics of the resulting models, particularly pertaining to metapopulation persistence, and discuss some implications for conservation. We will end with a summary and discussion of our findings.

\subsection{THE LEVINS MODEL}

The classical Levins model is generally written in terms of the following differential equation

$$
\frac{d p}{d t}=c p(1-p)-m p
$$


where $p$ is the fraction of occupied patches, $c$ is the colonization rate of empty patches, and $m$ is the extinction rate of occupied patches. Note that the colonization term $c p(1-p)$ is proportional to both the occupied patches $(p)$ and the empty patches $(1-p)$, as we mentioned above.

Defining $\beta:=c / m,(8.1)$ has the nontrivial equilibrium,

$$
p^{*}=1-\frac{1}{\beta}=: K .
$$

We call this $K$ because of its interpretation as the metapopulation carrying capacity (Amarasekare, 1998b). This equilibrium is stable as long as $\beta>1$ (if $\beta \leq 1$ the trivial equilibrium $p^{*}=0$ is stable).

To enable comparison with its stochastic version (see below) the Levins model has also been written in terms of the number of occupied patches (Etienne, 2002; Etienne and Nagelkerke, 2002; Harding and McNamara, 2002),

$$
\frac{d n}{d t}=c^{\prime} n(N-n)-m n
$$

where $n$ is the number of occupied patches and $N$ is the total number of patches in the metapopulation, so $p=n / N$. The colonization parameters $c^{\prime}$ and $c$ are related to one another by $c=c^{\prime} N$. The non-trivial equilibrium is, obviously, $n^{*}=N-m / c^{\prime}$.

\subsection{THE PATCH OCCUPANCY MODEL}

The Levins model is a special case of a family of models, called patch occupancy models. A patch occupancy model assumes that patches can be either occupied or empty and describes the dynamics of the number of occupied patches. There are deterministic and stochastic patch occupancy models, formulated in either continuous or discrete time. We will describe each of the four possible combinations. Because the Levins model is a patch occupancy model, there are also stochastic and discrete-time analogues of the Levins model. Treating the Levins model as a special case of a more general model clarifies the interpretation of the Levins model which may otherwise lead to inconsistencies, as we will see below.

The deterministic model in continuous time

The deterministic continuous-time patch occupancy model was presented by Gotelli and Kelley (1993) as

$$
\frac{d p}{d t}=C(p)(1-p)-M(p) p
$$


with $C(p)=c p$ and $M(p)=m$. Again $p$ is the fraction of occupied patches. In (8.4) it is clear that $p$ can be interpreted as the probability of a patch being occupied (Etienne, 2002; Etienne and Nagelkerke, 2002), although this was not explicitly mentioned by Gotelli and Kelley (1993). If the patch is empty (probability $1-p$ ), then it becomes occupied at rate $C(p)$ and when it is occupied (probability $p$ ), it becomes empty at rate $M(p)$.

When written in terms of the number of occupied patches, (8.4) becomes (Etienne, 2002; Harding and McNamara, 2002)

$$
\frac{d n}{d t}=C(n)(N-n)-M(n) n \text {. }
$$

The Levins model has $C(n)=c^{\prime} n$ and $M(n)=m$.

The stochastic model in continuous time

The general formulation of the stochastic continuous-time patch occupancy model describes the probability $P_{n}$ of $n$ patches (out of $N$ patches) being occupied, for all $0 \leq n \leq N$ :

$$
\begin{aligned}
\frac{d P_{n}}{d t}= & C(n-1)(N-(n-1)) P_{n-1} \\
& -[M(n) n+C(n)(N-n)] P_{n}+M(n)(n+1) P_{n+1}
\end{aligned}
$$

with $P_{n}=0$ for $n<0$ and $n>N$.

The model described by (8.6) is a Markov model (Frank and Wissel, 1998; Ovaskainen, 2001; Etienne, 2002; Etienne and Nagelkerke, 2002), so properties of Markovian models can be used to analyse the model. For example, when the model is written in matrix notation, the resulting matrix has the property that its second largest (in absolute value) eigenvalue is a measure of the expected time to extinction of the metapopulation when started in the quasi-stationary state. This quasi-stationary state is the left eigenvector corresponding to this second largest eigenvalue. The elements $i=2, \ldots, N$ of this eigenvector give the probability of $i-1$ patches being occupied, conditional on non-extinction of the metapopulation. The quasi-stationary state is therefore a probability distribution over all possible states; it is the probability distribution reached when the system has been undisturbed for some time, and can thus be considered a pseudo-equilibrium (it cannot be a real equilibrium, because the only real equilibrium is metapopulation extinction).

Formula (8.6) is also a special case of the stochastic birth-death model (Goel and Richter-Dyn, 1974),

$$
\frac{d P_{n}}{d t}=b_{n-1} P_{n-1}-\left[b_{n}+d_{n}\right] P_{n}+d_{n} P_{n+1}
$$

where $b_{n}$ and $d_{n}$ are the birth and death rates, respectively. With $b_{n}=C(n)(N-n)$ and $d_{n}=M(n) n$ we retrieve (8.6). Being a birth-death model, 
(8.6) possesses the convenient property that the expected time to absorption (i.e. extinction), when starting with $n_{0}$ patches occupied, is given by (Goel and Richter-Dyn, 1974):

$$
T_{\text {ext }}\left(n_{0}\right)=\sum_{i=1}^{n_{0}} \frac{1}{d_{i}}+\sum_{j=i+1}^{N}\left(\frac{1}{d_{j}} \prod_{k=i}^{j-1} \frac{b_{k}}{d_{k}}\right)
$$

This formula provides an alternative way of calculating the expected time to metapopulation extinction, when starting in the quasi-stationary state (Etienne and Nagelkerke, 2002).

The deterministic continuous-time model is a limiting case of the stochastic continuous-time model. We will not go into the appropriate limit here, but refer to Etienne (2002) for more details.

The stochastic continuous-time analogue of the Levins model is obtained from the stochastic continuous-time occupancy model by setting $C(n)=c^{\prime} n$ and $M(n)=m$.

The deterministic model in discrete time

The discrete-time analogue of (8.4) is given by the difference equation

$$
n_{t+\Delta t}=n_{t}+C\left(n_{t}, \Delta t\right)\left(N-n_{t}\right)-M\left(n_{t}, \Delta t\right) n_{t}
$$

where $n_{t}$ is the number of occupied patches at time $t, M\left(n_{t}, \Delta t\right)$ is the probability of extinction of a local population in one time-step of length $\Delta t$, and $C\left(n_{t}, \Delta t\right)$ is the probability that an empty patch will be colonized. We will give some examples of $C\left(n_{t}, \Delta t\right)$ below.

If we assume that

$$
\begin{gathered}
\lim _{\Delta \downarrow 0} \frac{C\left(n_{t}, \Delta t\right)}{\Delta t}=C(n) \\
\lim _{\Delta \downarrow t} \frac{M\left(n_{t}, \Delta t\right)}{\Delta t}=M(n)
\end{gathered}
$$

then (8.9) reduces to the continuous-time model

$$
\begin{aligned}
& \frac{d n}{d t}=\lim _{\Delta \downarrow t} \frac{n_{t+\Delta t}-n_{t}}{\Delta t}= \\
& =\lim _{\Delta \downarrow 0} \frac{C\left(n_{t}, \Delta t\right)\left(N-n_{t}\right)-M\left(n_{t}, \Delta t\right) n_{t}}{\Delta t}= \\
& =\left(N-n_{t}\right) \lim _{\Delta \downarrow 0} \frac{C\left(n_{t}, \Delta t\right)}{\Delta t}-n_{t} \lim _{\Delta \downarrow 0} \frac{M\left(n_{t}, \Delta t\right)}{\Delta t}= \\
& =C(n)(N-n)-M(n) n
\end{aligned}
$$

where in the last expression we have dropped the subscript $t$ to indicate that we are dealing with a continuous-time formulation. We use this notation throughout this paper. 
There are usually several functions for $C$ and $M$ that satisfy (8.8.10a) and (8.8.10b), because they only need to behave similarly near $\Delta t=0$. Therefore, there are several discrete-time analogues of the continuous-time patch occupancy model, and hence there are several discrete-time analogues of the original (deterministic continuous-time) Levins model as well.

The stochastic model in discrete time

In discrete time multiple extinctions and colonizations per time step are plausible. Suppose that the probability of a local population of becoming extinct in one time-step of length $\Delta t$ is $M(n, \Delta t)$ and that the probability of an empty patch of becoming occupied is $C(n, \Delta t)$ if there are $n$ occupied patches. This is fully analogous to the deterministic model in discrete time. The transition probability $P_{k l}$ to move from $k$ occupied patches to $l$ occupied patches in one time-step is then given by (see Gyllenberg and Silvestrov, 1994)

$$
\begin{aligned}
P_{k l}= & \sum_{i=0}^{\min (k, t)}\left(\begin{array}{c}
k \\
i
\end{array}\right) M(k, \Delta t)^{k-i} . \\
& {[1-M(k, \Delta t)]^{l}\left(\begin{array}{c}
N-i \\
l-i
\end{array}\right) C(k, \Delta t)^{L-i}[1-C(k, \Delta t)]^{N-l} . }
\end{aligned}
$$

The $P_{k l}$ form a matrix, of which the second largest eigenvalue is a measure of the metapopulation extinction time, similar to the continuous-time model. In (8.12), extinctions and colonizations can occur simultaneously. There is also a version where there is an extinction phase followed by a colonization phase (Akçakaya and Ginzburg, 1991; Day and Possingham, 1995). We obtain the corresponding formula for $P_{k l}$ by replacing $C(k, \Delta t)$ in (8.12) by $C(i, \Delta t)$.

We refrain from describing the transition from the stochastic discrete-time model to the stochastic continuous-time model, because this is more complicated and because we will not be using it in this paper.

\subsection{THE FOUNDER EFFECT}

In the introduction we defined the founder effect as the effect that colonization may be hampered by sheer stochasticity: even if the growth rate is positive, stochastic fluctuations in birth and death rates may prevent colonization. We will first incorporate the founder effect into the deterministic discrete-time patch occupancy model and then take the appropriate limit to obtain the continuous-time model. Subsequently, we will present a direct way of incorporating the founder effect in the continuous-time model. This gives a different model, but this model also has a different interpretation. We conclude the section with a more detailed model of the founder effect where 
stochasticity is not only present in the establishment of a new population, but already in reaching the destination patch.

\section{The deterministic model in discrete time}

In the Levins model the mechanism of colonization is not modelled explicitly. Here, we attempt to find a more mechanistic basis for the colonization term in the Levins model. This is most comprehensibly achieved in the discrete-time model (8.9). Colonization may be described by a cumulative-hit model. In this model, arrival of a disperser does not necessarily imply colonization, but the more dispersers arrive during the time interval $\Delta t$, the larger the probability of colonization is. The probability $C\left(n_{t}, \Delta t\right)$ of an empty patch being colonized during the time interval $\Delta t$ thus increases with $\Delta t$, because the number of dispersers arriving at the patch increases with $\Delta t$.

Assume that each patch produces $L(\Delta t)$ dispersers that immigrate into any patch, empty or occupied with probability $p_{\mathrm{d}}(\Delta t)$ (d of dispersal). Hence, each patch receives

$$
I\left(n_{t}, \Delta t\right)=\frac{L(\Delta t) p_{\mathrm{d}}(\Delta t) n_{t}}{N}
$$

dispersers during the time interval $\Delta t$; note that dispersers from all occupied patches are added together. We further assume that these dispersers arrive simultaneously, which is fairly realistic for species with a short dispersal period, and that they reproduce asexually. Local population dynamics are governed by a birth-death model (Goel and Richter-Dyn, 1974). The colonization probability, starting from $I\left(n_{t}, \Delta t\right)$ immigrants, is given by

$$
C\left(n_{t}, \Delta t\right)=1-\left(\frac{1}{R_{0}}\right)^{I\left(n_{t} \Delta t\right)}, R_{0}>1
$$

where $R_{0}$ is the basic reproduction number of the population, that is, the expected number of off-spring of each founder of the colony (Goel and Richter-Dyn, 1974). Because $C\left(n_{t}, \Delta t\right)<1$, the arrival of dispersers at an empty patch does not guarantee colonization, even if $R_{0}>1$, although unsuccessful colonization is very unlikely if there are many dispersers. This is the founder effect or establishment effect (Lande et al., 1998).

Let us now insert (8.14) into (8.9). Furthermore, we assume that the extinction probability does not depend on the number of occupied patches, so $M\left(n_{t}, \Delta t\right)=M(\Delta t)$. We will also assume that condition (8.8.10b) holds. Because our interest in this paper lies in the colonization process, this assumption will be used throughout the rest of this paper. Because

$$
\frac{d^{2}}{d n_{t}^{2}} C\left(n_{t}, \Delta t\right)\left(N-n_{t}\right)<0
$$


for all $\underline{n}_{t}<N$, the condition for a stable equilibrium is found to be (see also Figure 8.1$)$ :

$$
\frac{L(\Delta t) p_{\mathrm{d}}(\Delta t) \ln R_{0}}{M(\Delta t)}>1
$$

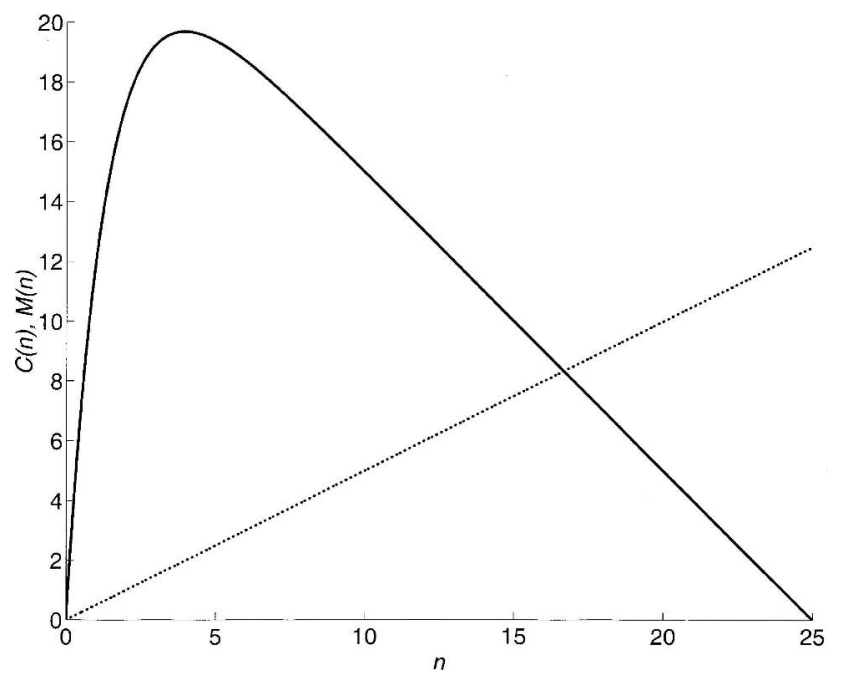

Figure 8.1. The contributions of colonization $\left(C\left(n_{t}, \Delta t\right)\left(N-n_{t}\right)\right.$, solid curve $)$ and extinction $\left(M(\Delta t) n_{t}\right.$, dotted curve) in the deterministic discrete-time Levins model, that is, (8.9) with $C\left(n_{t}, \Delta t\right)$ given by (8.17) and $M\left(n_{t}, \Delta t\right)=M(\Delta t)$. The equilibria are at the intersection points of these curves. Because the contribution of colonization always has a negative second derivative, a non-trivial equilibrium can only exist if the colonization contribution has a larger slope at the origin than the extinction contribution. In this figure we used the numerical values $N=25, s(\Delta t)=0.5$ and $M(\Delta t)=0.5$.

We would like to point out here that a similar condition would have been obtained without the birth-death model, and even without the cumulative-hit hypothesis; a one-hit model (arrival of at least one disperser at an empty patch entails colonization) may also apply. Suppose that during a time interval of length $\Delta t$ each occupied patch can colonize an empty patch with probability $s(\Delta t)$. This probability, which by definition does not depend on $n_{t}$, can represent the probability that at least one disperser will reach the empty patch (which is sufficient for colonization under the one-hit hypothesis) or the probability that the dispersers that arrive at the empty patch establish a population (as we assumed above), or both. The colonization probability is given by

$$
C\left(n_{t}, \Delta t\right)=1-(1-s(\Delta t))^{n_{t}}
$$


and this is mathematically equivalent to (8.17) if

$$
s(\Delta t)=1-\left(\frac{1}{R_{0}}\right)^{\frac{L(\Delta t) p_{\mathrm{d}}(\Delta t)}{N}} .
$$

Note that indeed $s(\Delta t)$ does not depend on $n_{t}$. With (8.17), the condition for a stable nontrivial equilibrium reads

$$
-\frac{N \ln (1-s(\Delta t))}{M(\Delta t)}>1 .
$$

The deterministic model: from discrete time to continuous time

We remarked above that under certain conditions the discrete-time Levins model reduces to the classical continuous-time Levins model. The condition for the colonization term, (8.8.10a), where $C(n)=c^{\prime} n$ and $M(n)=m$ for the Levins model, translates to the following expression in our model with (8.17):

$$
\lim _{\Delta \downarrow 0} \frac{s(\Delta t)}{\Delta t}=c^{\prime}
$$

where $c^{\prime}$ is a constant. For the cumulative-hit model, this in turn translates to

$$
\lim _{\Delta \downarrow t} \frac{I\left(n_{t}, \Delta t\right)}{\Delta t}=c^{\prime \prime}
$$

with $c^{\prime \prime}$ another constant. With (8.20) condition (8.8.10a) is indeed satisfied, because

$$
\begin{aligned}
& \lim _{\Delta \downarrow t} \frac{C\left(n_{t}, \Delta t\right)}{\Delta t}=\lim _{\Delta \downarrow 0} \frac{1-(1-s(\Delta t))^{n_{t}}}{\Delta t} \\
& =\lim _{\Delta \downarrow t} \frac{\left.n_{t}(1-s(\Delta t))^{n_{t}-1} \frac{d s(x)}{d x}\right|_{x=\Delta t}}{1}=c^{\prime} n_{t}
\end{aligned}
$$

Thus the original Levins model is recovered. Hence, the colonization term in the Levins model describes the founder effect.

The deterministic model in continuous time

Let us now look at an attempt to incorporate the founder effect in the deterministic continuous-time model directly (Etienne et al., 2002a). We adopt the interpretation of $c^{\prime}$ as the product of the rate $c_{\text {out }}$ at which dispersers are produced by an occupied patch and the probability $c_{\text {in }}$ with which a disperser successfully colonizes a patch (Frank and Wissel, 1998; Etienne and Heesterbeek, 2000; Ovaskainen and Hanski, 2001). The founder effect must evidently be inserted into $c_{\text {in }}$. Suppose, for instance, that $c_{\text {in }}=c_{\text {out }} n /\left(c_{\text {out }} n+a\right)$ for some parameter $a$, a formula that is similar to (8.14) and seems a 
reasonable choice to model the founder effect phenomenologically (Etienne $e t$ al., 2002a). It says that $c_{\text {in }}$ is a saturating function of the number of dispersers produced by all occupied patches per unit of time, $c_{\text {out }} n$. Note that this is different from the assumptions that led to (8.17), not just because a different saturating function is taken, but particularly because the argument of the function is the rate of dispersers produced instead of a number within a time window. The model becomes (Etienne et al., 2002a)

$$
\frac{d n}{d t}=c_{\text {out }} n \frac{c_{\text {out }} n}{c_{\text {out }} n+a}(N-n)-m n
$$

which shows entirely different dynamics than the Levins model (8.3). In fact, it is mathematically similar to the Levins model with Allee effect that we will construct below.

The difficulty with (8.23) is that the use of saturating functions in continuous-time models is tricky, because they often seem to rely on discretetime considerations, as we will show below for the Allee effect. In the current case, it is unclear what the mechanistic basis is for the saturating function for $c_{\text {in }}$, and therefore it is unclear whether it really describes the founder effect as we understand it. We expect $c_{\text {in }}$ to become constant for large $c_{\text {out }} n$, because for large $c_{\text {out }} n$ we no longer expect to find a founder effect, but it is not evident what the behavior for small $c_{\text {out }} n$ should be. Our expressions (8.14) and (8.17) are less phenomenological than the expression for $c_{\text {in }}$, because they are based on probabilistic arguments of whether a set of dispersers can establish a population.

In summary, the founder effect is contained in the Levins model as our transition from discrete to continuous time has shown, and the model (8.23) does not describe the founder effect (but the Allee effect, see below).

Stochasticity in dispersal: colonization average vs. average colonization in the founder effect

Until now we have assumed that a fixed number of dispersers, $L(\Delta t) p_{\mathrm{d}}(\Delta t) n_{t} / N$, arrives at an empty patch during the time interval $\Delta t$ upon which colonization may or may not occur. Hence, the number of arriving dispersers is modelled deterministically. If, instead, we take the number of dispersers leaving the occupied patch to be a fixed number, say $L(\Delta t)$, and if we let the probability of reaching a patch be governed by a binomial distribution with parameter $p_{\mathrm{d}}(\Delta t)$, then we only have on average

$$
\bar{I}\left(n_{t}, \Delta t\right)=\frac{L(\Delta t) p_{\mathrm{d}}(\Delta t) n_{t}}{N} .
$$

By inserting this expression into (8.14), we would effectively use the colonization probability of the average number of arriving dispersers: 


$$
C\left(\bar{I}\left(n_{t}, \Delta t\right)\right)=1-\left(\frac{1}{R_{0}}\right)^{\frac{L(\Delta t) p_{d}(\Delta t) n_{t}}{N}} .
$$

This is incorrect. We should use the average colonization probability instead:

$$
\begin{aligned}
\overline{C\left(I\left(n_{t}, \Delta t\right)\right)} & =\sum_{j=0}^{\frac{L(\Delta t) n_{t}}{N}}\left[1-\left(\frac{1}{R_{0}}\right)^{j}\right]\left(\begin{array}{c}
\frac{L(\Delta t) n_{t}}{N} \\
j
\end{array}\right)\left[p_{\mathrm{d}}(\Delta t)\right]^{j}\left[1-p_{\mathrm{d}}(\Delta t)\right]^{\frac{L(\Delta t) n_{t}}{N}-j} \\
& =1-\left[1-p_{\mathrm{d}}(\Delta t)\left(1-\frac{1}{R_{0}}\right)\right]^{\frac{L(\Delta t) n_{t}}{N}} .
\end{aligned}
$$

Equations (8.25) and (8.26) are not equal. Figure 8.2 shows them for $L(\Delta t) n_{t} / N=100$ and $R_{0}=3$. Using (8.25) therefore leads to a higher colonization probability.

In the following sections, we will adhere to the original model with a deterministic (fixed) number of dispersers arriving at an empty patch, because it is qualitatively equivalent to, but much simpler than the more detailed model where the number of dispersers arriving at an empty patch is a stochastic variable. As for quantitative differences, we checked these for several parameter settings and found that they are never very large.

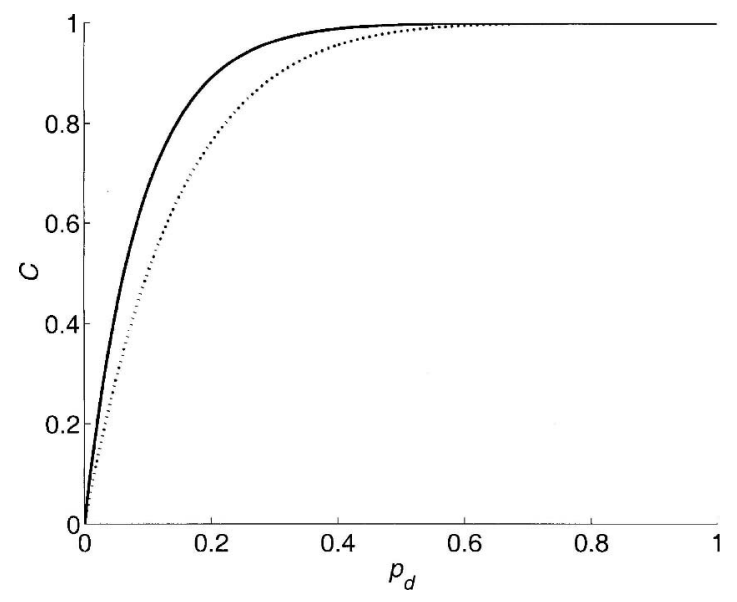

Figure 8.2. The colonization function of the average number of immigrants (solid curve) and the average colonization function of the number of immigrants (dotted line) plotted against the probability of successful dispersal $p_{\mathrm{d}}$. Taking the average at the level of arriving immigrants thus leads to an overestimate of the colonization function. Numerical values of the other parameters are $L(\Delta t) n_{t} / N=100$ and $R_{0}=3$. 


\subsection{THE ALLEE EFFECT}

The Allee effect differs from the founder effect in that the Allee effect denotes a reduced or negative deterministic growth rate at small population sizes, whereas the founder effect is a purely stochastic phenomenon, most active at small population sizes, that occurs even with an unreduced positive growth rate. We will incorporate the Allee effect (at the population level, not at the metapopulation level as in Amarasekare, 1998b) in the deterministic discrete-time model, and then take the appropriate limit to get the analogous continuous-time model. Because this yields a rather undesirable result, we will then start from a continuous-time formulation and convert this back to discrete-time. In all cases we will study metapopulation dynamics. To gain insight into the threshold phenomenon exhibited in the deterministic models, we move to a stochastic formulation. We end by examining the Allee effect in conjunction with the patch preference effect, because we think that these will often occur together, and because they may represent opposing forces making the outcome less obvious.

\section{The deterministic model in discrete time}

The founder effect typically represents the case where the colonization probability $C\left(n_{t}, \Delta t\right)$ is linear in the number of occupied patches $n_{t}$ when $s(\Delta t)$ is small. This means that contributions from different patches are simply additive. When the Allee effect is active, the colonization probability behaves differently. There may be a minimum number of immigrants necessary for the population to grow and thus for colonization to occur or $C\left(n_{t}, \Delta t\right)$ may initially be quadratic in the number of immigrants (or the number of occupied patches producing dispersers). Instead of a detailed model of colonization for a population suffering from the Allee effect, we consider a more phenomenological model, again in discrete time because of its easier interpretation:

$$
C\left(n_{t}, \Delta t\right)=\frac{I^{2}\left(n_{t}, \Delta t\right)}{I^{2}\left(n_{t}, \Delta t\right)+y^{2}}
$$

where $y$ is some constant, measuring the strength of the Allee effect. Thus, colonization as a function of the number of immigrants has a sigmoidal shape. Formula (8.27) is not just an ad hoc choice; it is used in the well-known incidence function model of Hanski (1994).

Let us again assume that the number of immigrants $I\left(n_{t}, \Delta t\right)$ entering an empty patch during time interval $\Delta t$ is determined by the number of dispersers that are produced by each occupied patch, $L(\Delta t)$, and by the probability that each disperser reaches the empty patch, that is, we use (8.13). We can rewrite (8.27) as 


$$
C\left(n_{t}, \Delta t\right)=\frac{n_{t}^{2}}{n_{t}^{2}+y^{\prime 2}(\Delta t)}
$$

where $y^{\prime}(\Delta t)=y N /\left(L(\Delta t) p_{\mathrm{d}}(\Delta t)\right)$. $n^{*}$ :

Inserting this into (8.9) leads to the following equation for the equilibrium

$$
\frac{n^{* 2}}{n^{*^{2}}+y^{\prime 2}(\Delta t)}\left(N-n^{*}\right)=M(\Delta t) n^{*}
$$

This results in three equilibria, dropping the dependence on $\Delta t$ for notational convenience:

$$
\begin{aligned}
n_{0}^{*} & =0 \\
n_{-}^{*} & =\frac{N}{1+M}\left(\frac{1}{2}-\frac{1}{2} \sqrt{1-4 \frac{M y^{\prime 2}(1+M)}{N^{2}}}\right) \\
& =\frac{N}{1+M}\left(\frac{1}{2}-\frac{1}{2} \sqrt{1-4 M y^{\prime 2}(1+M)}\right) \\
n_{+}^{*} & =\frac{N}{1+M}\left(\frac{1}{2}+\frac{1}{2} \sqrt{1-4 \frac{M y^{\prime 2}(1+M)}{N^{2}}}\right) \\
& =\frac{N}{1+M}\left(\frac{1}{2}+\frac{1}{2} \sqrt{1-4 M y^{\prime 2}(1+M)}\right)
\end{aligned}
$$

where we have defined $y^{\prime \prime}=y^{\prime} / N=y /\left(L p_{\mathrm{d}}\right)$. Of these solutions, $n_{-}{ }^{*}$ is unstable and forms the separatrix between the other two, stable, solutions. This means that, when the system is started below the separatrix, the metapopulation will become extinct, and when started above the separatrix, it will converge to the nontrivial equilibrium $n_{+}{ }^{*}$. See Figure 8.3.

Figure 8.3A shows the catastrophic consequences of increased dispersal resistance due to (rail) roads and the like (increased dispersal resistance means a reduction of the probability of reaching a patch $p_{\mathrm{d}}$ and hence an increase in $\left.y^{\prime \prime}\right)$. If this dispersal resistance grows gradually (for example, because there is a gradual increase of the amount of cars using the road), then $p^{*}$ may seem to be hardly affected, until suddenly the metapopulation disappears (in Figure $8.3 \mathrm{~A}$ this happens for $y^{\prime \prime} \approx 2.88$ ). Figure $8.3 \mathrm{~B}$ shows similar behaviour for increases in the probability of local extinction, for example due to habitat deterioration.

Harding and McNamara (2002) also used (8.27), but they did so in the continuous-time model (8.5), that is, they set $C(n)=\alpha^{2}(n) /\left(\alpha^{2}(n)+y^{2}\right)$, following Hanski (1994). However, Hanski's (1994) model is a discrete-time model, and $C(n)$ is the probability of colonization per time-step, whereas a continuous-time model requires a probability rate. They do define $\alpha$ as the 
immigration rate, which is correctly used in the Levins model where they have $C(n) \sim \alpha(n)$, but this does not make $C(n)$ a rate in this case. Specifically, $C(n)$ cannot become greater than unity in this formulation (because it is in fact a probability), but the colonization rate may surely become much greater than unity; it usually becomes infinite when the immigration rate becomes infinite. This is not just a matter of a scaling factor. Although Harding and McNamara (2002) may have omitted a constant to simplify their expressions, addition of a constant, that is, using $C(n)=k \alpha^{2}(n) /\left(\alpha^{2}(n)+y^{2}\right)$ instead where $k$ is a constant with the dimension of rate, does not resolve the issue. The colonization rate is then explicitly allowed to become larger than unity, but an infinite immigration rate still does not result in an infinite colonization rate. Of course, one can have a model where an infinite immigration rate does not automatically correspond to an infinite colonization rate, but then one is trying to model a different phenomenon, not the Allee effect. Thus, apparently it is not straightforward to incorporate the Allee effect into the continuous-time Levins model as it seems, so our attempt seems well justified.

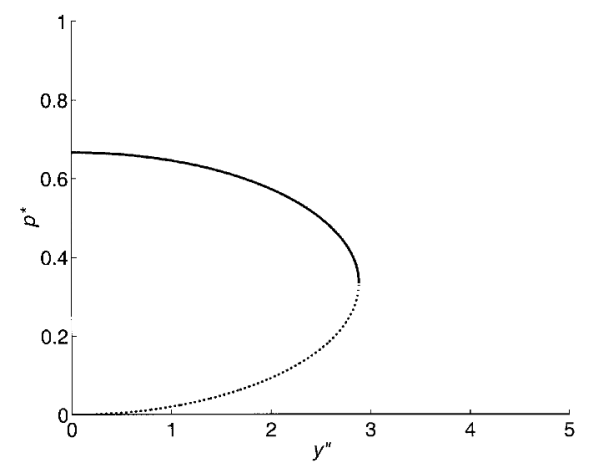

A

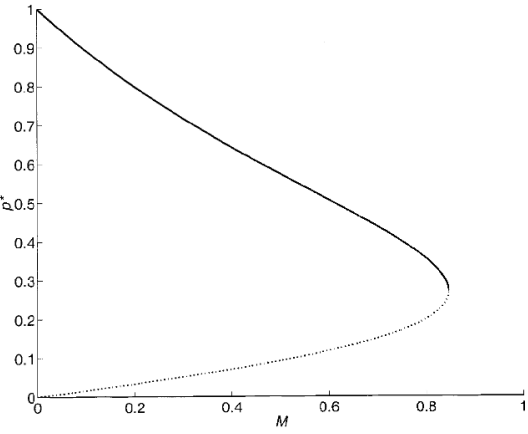

B

Figure 8.3. Bifurcation diagrams of the discrete-time model (8.9) with Allee effect as in (8.27) and $M\left(n_{t}, \Delta t\right)=M(\Delta t)=M$. The solid curves represent the stable equilibria, the dotted line represents the unstable equilibrium. A fold bifurcation occurs where the unstable and stable branches meet. A: The bifurcation parameter is $y^{\prime \prime} ; M=0.5$. $\mathrm{B}$ : The bifurcation parameter is $M ; y^{\prime \prime}=0.4$.

The deterministic model: from discrete to continuous time and back

Above we noted that the Levins model with founder effect is just the Levins model if (8.21) is satisfied. Now, let us examine what happens if we use this same condition in the Levins model with Allee effect. Using (8.27): 


$$
\begin{aligned}
& \lim _{\Delta \downarrow t} \frac{C\left(n_{t}, \Delta t\right)}{\Delta t}=\lim _{\Delta \downarrow t} \frac{\frac{I^{2}\left(n_{t}, \Delta t\right)}{I^{2}\left(n_{t}, \Delta t\right)+y^{2}}}{\Delta t}= \\
& \lim _{\Delta \downarrow t 0} \frac{\left.\left(I^{2}\left(n_{t}, \Delta t\right)+y^{2}\right) 2 I\left(n_{t}, \Delta t\right) \frac{d I(x)}{d x}\right|_{x=\Delta t}-\left.I^{2}\left(n_{t}, \Delta t\right) 2 I\left(n_{t}, \Delta t\right) \frac{d I(x)}{d x}\right|_{x=\Delta t}=0}{\left(I^{2}\left(n_{t}, \Delta t\right)+y^{2}\right)^{2}}=0
\end{aligned}
$$

because $I\left(n_{t}, 0\right)=0$. Hence, the conversion to a continuous-time model leads to a model in which the metapopulation cannot persist!

This is certainly an undesirable result, so the suggestion presents itself that we are dealing with an artefact inherent to our choice of (8.27). To examine this in more detail, we take a little detour. We start with the colonization term of the continuous-time Levins model in the Harding and McNamara (2002) framework, that is,

$$
C(n)=k \alpha n
$$

where $k$ is a positive constant and $\alpha$ the immigration rate, with the metapopulation dynamics governed by (8.5), so in effect we have $c^{\prime}=k \alpha$. This $C(n)$ represents the colonization probability per unit time. Consider a patch that is empty at $t=0$ and suppose that $n$ patches are occupied at time $t=0$ and that local extinction is impossible. The probability $p$ that the empty patch is colonized (and hence occupied) at time $t$ is described by

$$
\frac{d p}{d t}=C(n)(1-p)=k \alpha n(1-p) \text {. }
$$

The solution is readily found to be $p(t)=1-\exp (-k \alpha n t)$. Hence, after a time interval $\Delta t$ the probability that the patch is occupied is $p(\Delta t)=$ $1-\exp (-k \alpha n \Delta t)$. We can use this as the colonization probability in the discrete-time model (8.9), that is,

$$
C\left(n_{t}, \Delta t\right)=1-\exp \left(-k I\left(n_{t}, \Delta t\right)\right)
$$

with $I\left(n_{t}, \Delta t\right)=n \alpha \Delta t$. This equation is mathematically equivalent to (8.14) with $R_{0}=\exp (k)$. So, the continuous-time model with linear colonization rate corresponds to the discrete-time model with colonization probability that approaches its maximum of unity at an exponentially decreasing rate. The question now arises whether we can find a colonization formula in the continuous-time model that corresponds to a colonization probability in the discrete-time model with the sigmoidal shape that is so typical of the Allee effect. Evidently, (8.27) is surely not the only function with a sigmoidal shape.

Of this colonization formula in the continuous-time model we require that it is linear in the immigration rate $\alpha$ for large $\alpha$ and that it is quadratic in the immigration rate for small $\alpha$. Perhaps the simplest formula satisfying these requirements is 


$$
C(n)=k \frac{(\alpha n)^{2}}{\alpha n+\alpha_{0}}
$$

where $\alpha_{0}$ measures the strength of the Allee effect; for $\alpha_{0}=0$ we obtain the Levins model colonization term $C(n)=k \alpha n$. Using an argument similar to the one making the transition from (8.32) to (8.34), we find that equation (8.35) corresponds to a colonization probability in the discrete-time model

$$
C\left(n_{t}, \Delta t\right)=1-\exp \left(-k \frac{\left(\alpha n_{t}\right)^{2}}{\alpha n_{t}+\alpha_{0}} \Delta t\right) .
$$

The colonization rates and corresponding colonization probabilities are shown in Figure 8.4. We stress that although (8.35) is not a mechanistically derived model, its parameters have a biological meaning, which may be substituted by expressions that are derived from mechanistic submodels. Below, we will present an example of this.

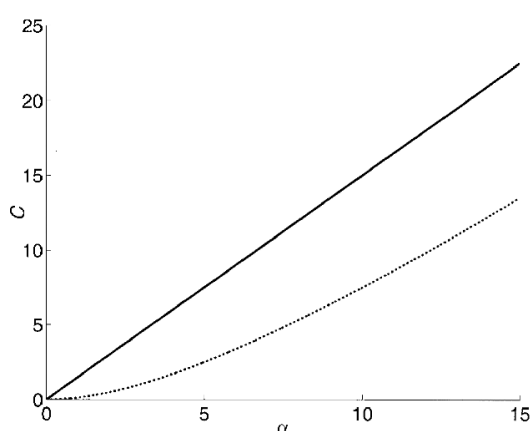

A

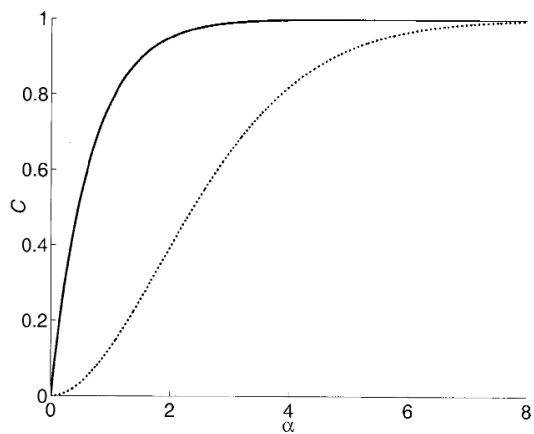

B

Figure 8.4. Colonization as a function of the immigration rate $\alpha$ for the Levins model with founder effect (equations (8.32) and (8.34), solid curves) and with Allee effect (equations (8.35) and (8.36), dotted curves) for continuous time (A) and for discrete time (B). Parameter values are $k=1.5$ and $\alpha_{0}=10$. We further set $n=1$.

The equilibrium number of patches for the discrete-time model with (8.36) is given by the solution of

$$
\left[1-\exp \left(-k \frac{\left(\alpha n^{*}\right)^{2}}{\alpha n^{*}+\alpha_{0}} \Delta t\right)\right]\left(N-n^{*}\right)=M n^{*} .
$$


In Figure 8.5 we have plotted the colonization and extinction terms. It can easily be seen that, in contrast to Figure 8.1, there may be two nontrivial equilibria, of which the larger one is stable. They coincide if

$$
\left.\frac{d}{d n}\left[1-\exp \left(-k \frac{(\alpha n)^{2}}{\alpha n+\alpha_{0}} \Delta t\right)\right](N-n)\right|_{n=n^{*}}=M
$$

which is again a fold bifurcation.

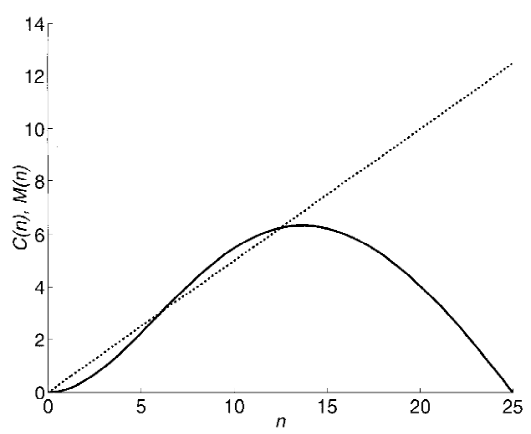

Figure 8.5. The colonization (solid curve) and extinction functions (dotted curve) in the deterministic discrete-time Levins model (8.9) with (8.36) and $M\left(n_{t}, \Delta t\right)=M(\Delta t)$. The equilibria are at the intersection points of these curves. There can be two nontrivial equilibria, the larger one of which is stable. In this figure we used the numerical values $N=25, \alpha_{0}=100, k=0.5, \Delta=1, \Delta t=1$, and $M(\Delta t)=0.5$.

Thus, this model behaves qualitatively similarly to the solution to (8.29). We conclude that (8.35) and (8.36) provide a consistent way to incorporate the Allee effect into the continuous-time and discrete-time Levins model respectively. We think that (8.36) is also a better formula than (8.27), because (8.36) does not lead to undesirable results when converted to its continuoustime analogue and it can be reduced to (8.34) by setting $\alpha_{0}=0$.

There is one subtlety in (8.36) that needs some attention. If we rewrite (8.36) in terms of the number of immigrants $I\left(n_{t}, \Delta t\right)=\alpha n_{t} \Delta t$, we obtain

$$
C\left(n_{t}, \Delta t\right)=1-\exp \left(-k \frac{I^{2}\left(n_{t}, \Delta t\right)}{I\left(n_{t}, \Delta t\right)+I_{0}(\Delta t)}\right)
$$

where $I_{0}(\Delta t)=\alpha_{0} \Delta t$. Hence, the strength of the Allee effect as measured by $I_{0}(\Delta t)$ depends on $\Delta t$. A longer time interval $\Delta t$ will therefore have a positive and a negative effect on the colonization probability: the number of arriving dispersers increases, but so does $I_{0}(\Delta t)$. The net increase is positive and linear in $\Delta t$ for small $\Delta t$, as it should be in order to obey (8.8.10a). 
When we compare the Allee effect model, (8.35), to the colonization term in (8.23), which was used by Etienne et al. (2002a) for the founder effect, we see that the colonization terms are identical, apart from the constant $k$. So, the results obtained by Etienne et al. (2002a) actually apply to the Levins model with (sigmoidal) Allee effect.

The stochastic model in continuous time

The bifurcation in the deterministic models can be used as a warning that small changes in parameters (such as $m$ ) may cause the nontrivial equilibrium to disappear suddenly, and thus metapopulation extinction. If these changes are really so abrupt, it is interesting to investigate if there are also abrupt changes in the stochastic model. To examine this, we will consider the continuous-time model (8.6) with Allee effect as modelled in (8.35). We choose the continuous-time model, merely because it allows for explicit analytical solutions. Results obtained with the discrete-time model are similar.

But let us first look at the deterministic counterpart, (8.5) and (8.35), for comparison. The equilibria are the solutions of

$$
k \frac{\left(\alpha n^{*}\right)^{2}}{\alpha n^{*}+\alpha_{0}}\left(N-n^{*}\right)=m n^{*}
$$

which are readily found to be (see also Etienne et al. 2002a)

$$
\begin{aligned}
& n_{0}^{*}=0 \\
& n_{-}^{*}=\frac{1}{2}\left(N-\frac{m}{c^{\prime}}\right)\left(1-\sqrt{1-4 \frac{\frac{m}{c^{\prime}} \frac{\alpha_{0}}{\alpha}}{\left(N-\frac{m}{c^{\prime}}\right)^{2}}}\right) \\
& n_{+}^{*}=\frac{1}{2}\left(N-\frac{m}{c^{\prime}}\right)\left(1+\sqrt{1-4 \frac{\frac{m}{c^{\prime}} \frac{\alpha_{0}}{\alpha}}{\left(N-\frac{m}{c^{\prime}}\right)^{2}}}\right)
\end{aligned}
$$

where $c^{\prime}:=k \alpha$ as before.

Returning to the stochastic model, for a system starting in the quasistationary state the expected time to metapopulation extinction and the expected occupancy conditional on non-extinction can be computed using the eigenvalues and eigenvectors of the matrix corresponding to (8.6), as we mentioned above. For systems starting in another state, e.g. all patches occupied, the expected metapopulation extinction time and the expected occupancy at some specified time can be calculated using (8.8) and the 
solution to (8.6) respectively. The mean occupancy at time $t=10$ of a metapopulation with all patches occupied at time $t=0$ is plotted in Figure 8.6A, together with the deterministic non-trivial equilibria. The results for the Levins model without Allee effect are also shown. We see that there is no abrupt change in the expected occupancy, although it does decrease faster than in the model without Allee effect. The expected time to metapopulation extinction, plotted in Figure 8.6B, behaves similarly. Neither does it make a difference if we use the expected occupancies and extinction times for systems starting in the quasi-stationary state or any other state (results not shown). As $N$ increases, the expected occupancy follows the deterministic equilibrium more closely and the expected extinction time increases more steeply with increasing immigration rate, but the bifurcation point is found at lower immigration rates. We therefore conclude that the Allee effect does have a detrimental effect on metapopulation persistence, but small parameter changes will not lead to sudden extinction when the number of patches is moderate. Only when there are many patches, the bifurcation of the deterministic model is reflected in the stochastic quantities of expected occupancy and extinction time, but the inviability region is then much smaller (Figure 8.7).

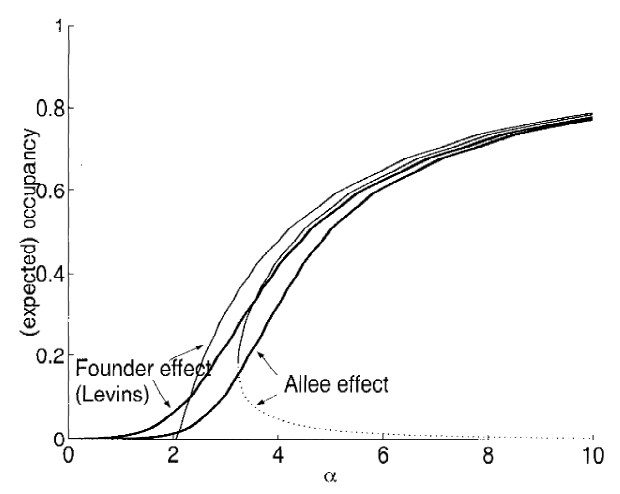

A

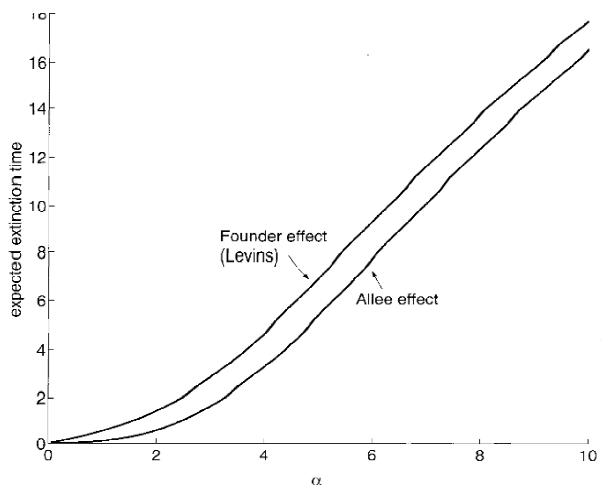

$\mathrm{B}$

Figure 8.6. The mean occupancy at time $t=10$ starting with all patches occupied (A) and the expected time to metapopulation extinction (B) for the Levins model with and without the Allee effect as a function of the immigration rate $\alpha$. In $\mathrm{A}$ the deterministic equilibrium values are also shown (thin lines). The values of the other parameters are $m=0.52, N=25, k=0.01$ and $\alpha_{0}=10$. 


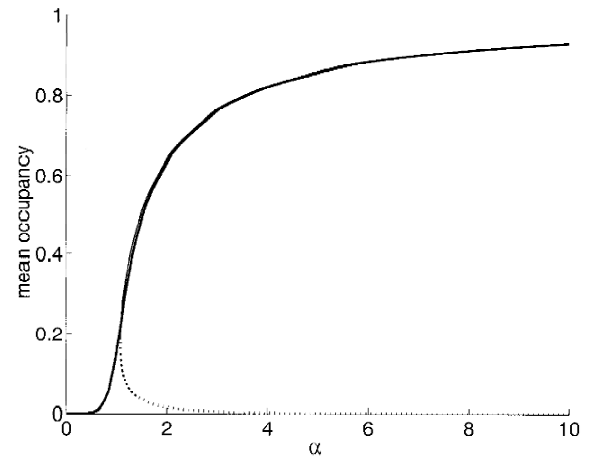

A

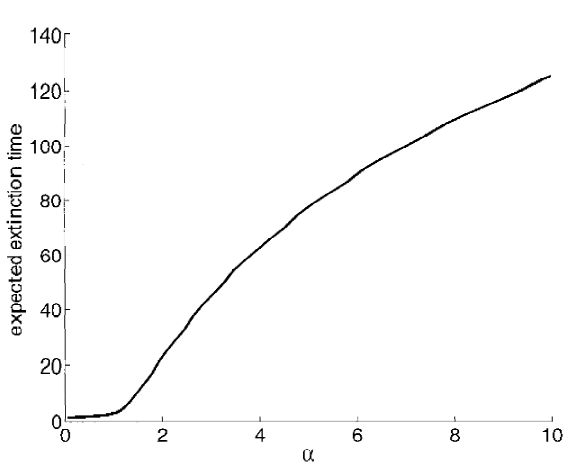

B

Figure 8.7. The mean occupancy at time $t=10$ starting with all patches occupied (A) and the expected time to metapopulation extinction (B) for the Levins model with Allee effect as a function of the immigration rate $\alpha$. In $\mathrm{A}$ the deterministic equilibrium values are also shown (thin lines). The number of patches is $N=75$. The values of the other parameters are the same as in Figure 8.6: $m=0.52, k=0.01$ and $\alpha_{0}=10$.

Overcoming the Allee effect: the patch preference effect

If dispersers are able to distinguish occupied from empty patches and have a preference for empty patches, the negative impact of the Allee effect may be partly nullified. We will take a different approach than Etienne (2000) in modelling the patch preference effect, because Etienne (2000) based his model on a mass action interpretation of the colonization term, which is much less transparent than our interpretation as outlined below (8.4). We take the discrete-time model (8.9) with (8.36), but we assume that, instead of (8.13), all immigrants go to empty patches, so the number of immigrants is given by

$$
I^{\prime}\left(n_{t}, \Delta t\right)=\frac{L(\Delta t) p_{\mathrm{d}}(\Delta t) n_{t}}{N-n_{t}} .
$$

With (8.42) the colonization probability becomes

$$
\begin{aligned}
& C\left(n_{t}, \Delta t\right)=1-\exp \left(-k \frac{I^{\prime 2}\left(n_{t}, \Delta t\right)}{I^{\prime}\left(n_{t}, \Delta t\right)+I_{0}(\Delta t)}\right)= \\
& =1-\exp \left(-k L(\Delta t) p_{\mathrm{d}}(\Delta t) \frac{\left(\frac{n_{t}}{N-n_{t}}\right)^{2}}{\frac{n_{t}}{N-n_{t}}+\frac{I_{0}(\Delta t)}{L(\Delta t) p_{\mathrm{d}}(\Delta t)}}\right) .
\end{aligned}
$$


The equilibria are the solutions of

$$
\left[1-\exp \left(-k^{\prime} \frac{\left(\frac{n^{*}}{N-n^{*}}\right)^{2}}{\frac{n^{*}}{N-n^{*}}+y^{\prime \prime}}\right)\right]\left(N-n^{*}\right)=M n^{*}
$$

where we defined $k^{\prime}:=k L(\Delta t) p_{\mathrm{d}}(\Delta t), y^{\prime \prime}:=I_{0}(\Delta t) /\left(L(\Delta t) p_{\mathrm{d}}(\Delta t)\right)$ and $M=M(\Delta t)$. There is no explicit expression for the solutions, except for $n_{0}{ }^{*}=0$ which is a stable equilibrium, but we do have explicit expressions for the bifurcation parameters $y^{\prime \prime}$ and $M$ :

$$
\begin{gathered}
y^{\prime \prime}=-\frac{n^{*}}{N-n^{*}}\left(1+\frac{k^{\prime} n^{*}}{\left.\left(N-n^{*}\right) \ln \left(\frac{N-n^{*}(1+M)}{N-n^{*}}\right)\right)}\right. \\
M=\left[1-\exp \left(-k^{\prime} \frac{\left(\frac{n^{*}}{N-n^{*}}\right)^{2}}{\frac{n^{*}}{N-n^{*}}+y^{\prime \prime}}\right)\right] \frac{N-n^{*}}{n^{*}} .
\end{gathered}
$$

The bifurcation diagrams are shown in Figure 8.8.

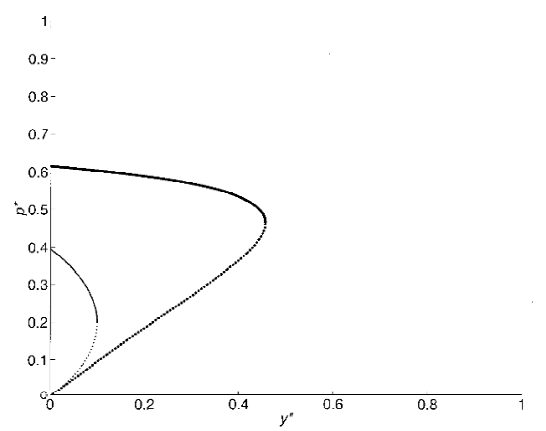

A

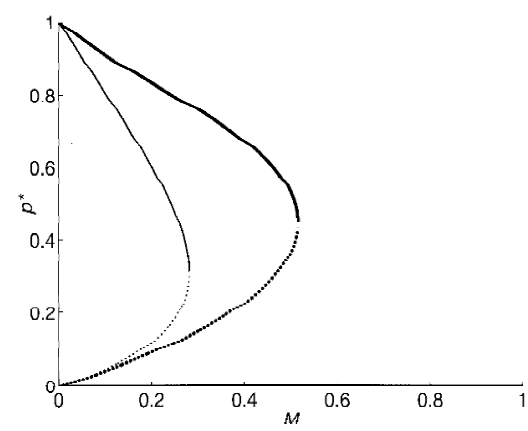

$\mathrm{B}$

Figure 8.8. Bifurcation diagrams of the discrete-time model (8.9) with Allee effect and patch reference effect as in (8.43) and $M\left(n_{t}, \Delta t\right)=M(\Delta t)=M$ (thick lines) and Allee effect only (thin lines). The solid curves represent the stable equilibria, the dotted line represents the unstable equilibrium. A fold bifurcation occurs where the unstable and stable branches meet. A. The bifurcation parameter is $y^{\prime \prime} ; M=0.5$. B. The bifurcation parameter is $M ; y^{\prime \prime}=0.4$. In both panels $k^{\prime}=1$. 
As expected, the equilibrium fraction of occupied patches in the model with Allee effect and patch preference effect is higher than the equilibrium fraction in the model with Allee effect but without patch preference effect. It is even higher than the model without Allee effect and patch preference effect (for which $y^{\prime \prime}=0$ ) for all values of $y^{\prime \prime}$ that allow a non-trivial equilibrium. In this sense we can say that patch preference can overcome the Allee effect. In other words: aggregation overcomes the Allee effect.

\subsection{DISCUSSION}

In this paper we have explicitly shown that the founder effect is contained in the Levins model. We also demonstrated how to consistently incorporate the Allee effect (acting at the population level) into the Levins model. We moved between discrete-time and continuous-time, deterministic and stochastic formulations of the metapopulation model to see how these formulations are related and to cast different lights on the founder and Allee effects. In these formulations, we prefer the interpretation of the patch occupancy as the probability of being occupied. This is equivalent to the patch occupancy in the mean-field approximation, but has the advantage that it can be consistently extended to cases in which this approximation no longer applies.

In our study of the founder and Allee effects we stressed the difference between the colonization term in the continuous-time and discrete-time formulations of the models, which, in spite of its apparent triviality, has led to some misunderstanding. The direct attempts of Etienne et al. (2002a) and Harding and McNamara (2002) to model the founder and Allee effects in a continuous-time model lead to models that describe something different from what they had in mind. This happens because they used, in their continuoustime models, saturating functions that are based on discrete-time considerations. Etienne et al. (2002a) attempted to model the founder effect, but they ended up with a model describing the Allee effect. Harding and McNamara (2002) attempted to model the Allee effect in a continuous-time model, but their results are actually valid for the discrete-time model (8.9). They did not notice that they were erroneous, because their results did not contradict their intuitions; Etienne et al. (2002a) considered the founder effect to be a special case of the Allee effect (with which we no longer agree), whereas Harding and McNamara (2002) still studied the Allee effect, but for a different type of model.

We demonstrated that under certain conditions the discrete-time model reduces to the continuous-time model when the appropriate limit is taken. The colonization function in the birth-death model (Goel and Richter-Dyn, 1974), representing the founder effect, satisfies these conditions, and hence the Levins model can be claimed to incorporate the founder effect, as has always been assumed. However, these conditions are not satisfied by the widely 
applied Hanski (1994) sigmoidal formulation considered to represent the Allee effect. On the contrary, this formulation leads to metapopulation extinction in all cases when transformed to the continuous-time model. We formulated a new function to describe the Allee effect that has the correct properties in both the discrete-time and the continuous-time formulations, and has the advantage over the function of Hanski (1994) that it reduces to the founder effect function when the parameter measuring the Allee effect is set to 0 . This comes at a price, though: the Allee effect is no longer associated solely with a threshold of the number of immigrants arriving in a certain time interval, but this threshold also depends on the length of the time interval. This is not biologically unreasonable: colonization is less successful when the time interval is longer while the number of immigrants remains the same, because in this case immigrants arrive at the patch in smaller groups less simultaneously, making the Allee effect potentially stronger.

Our new function for the Allee effect is, just as the one based on Hanski (1994), a purely phenomenological model of the Allee effect: no mechanistic basis has been provided. Incorporation of a phenomenological submodel in a mechanistic model need not be a problem at all, as this has been common practice with the rescue effect as well (Hanski, 1983; Hanski et al., 1996); in a broader population modeling context, the logistic growth model provides a well-known example. Still, for the rescue effect the underlying mechanism has been studied in more detail (Gyllenberg and Hanski, 1997; Etienne, 2002), so a more mechanistic approach is also recommended for the Allee effect.

Using our new function in the stochastic and deterministic formulations, we showed that the fold bifurcation in the deterministic model should not be given the interpretation that a small change in parameter values may cause an abrupt change in metapopulation persistence, that is, sudden metapopulation extinction, if the network contains a moderate number of patches. This is readily explained. In the deterministic model the equilibrium suddenly (dis)appears when the bifurcation parameter passes the bifurcation point, but the stability of the equilibrium changes continuously, vanishing at the bifurcation point (because the stable and unstable branch meet at the fold bifurcation). Hence, the attracting force of the non-trivial equilibrium changes gradually. Only when the number of patches is large, are there sharp changes in the attracting force, so that the stochastic model increasingly resembles the deterministic model as the number of patches increase. This is indeed what we observed.

To reduce the impact of the Allee effect, one need not increase the immigration rate per se. We showed that increasing patch preference for empty patches also diminishes the Allee effect. Preference for empty patches can be a natural phenomenon, for example it is plausible for territorial species. But preference for empty patches can also be enforced by (temporarily) closing corridors connecting occupied patches, thus redirecting migration to empty patches. However, preference for empty patches may also decrease the 
benefits of the rescue effect (Etienne, 2000). The net result for metapopulation persistence depends on the relative strengths of the effects. This deserves further study.

We have looked at the founder and Allee effects in the simple patch occupancy model that ignores population size. It seems more appropriate to study the Allee effect with an individual-based model or a model where population sizes are explicitly taken into account, instead of in a patch occupancy model where individuals are only implicit. These individual-based or size-structured metapopulation models are, however, usually much more complicated and thus more difficult to comprehend. A simple model as the patch occupancy model provides a strong metaphor with which predictions can be obtained fairly quickly. These predictions can (and must) then be tested in more complicated models, and if the predictions of these models are different, one must critically examine the structure of these models to find out why. In this way, one can systematically gain more insight into what processes are relevant. This is the primary value of simple models. For the very same reason, the rescue effect, the patch preference effect and the like have also been studied in the simple patch occupancy model (see the introduction for references). The secondary value of simple models is that they can be extended to more realistic (and therefore usually more complicated) models. It is then of great importance that the mathematical description of the simple model exactly corresponds with one's assumptions. If, for example, the models of Etienne et al. (2002a) and Harding and McNamara (2002) were extended to include competition or predator-prey interactions (see the introduction for references on how to do so), the results may no longer correspond with their initial assumptions.

Analysis of the dynamics (or rather the equilibria) of the patch occupancy model with founder and Allee effect did not yield results that have not been obtained earlier with more complicated models. However, this does not make our model superfluous. On the contrary, it means that for many purposes, our simple model suffices as a description of the founder and Allee effects.

\section{REFERENCES}

Akçakaya, H.R. and L.R. Ginzburg (1991). Ecological risk analysis for single and multiple populations. In: Seitz, A. and V. Loeschcke (Eds). Species conservation: a population-biological approach. Birkhäuser Verlag, Basel, Switzerland. pp. 73-85.

Allee, W.C. (1931). Animal aggregations, a study in general sociology. University of Chicago Press, Chicago, USA.

Alonso, D. and A. McKane (2002). Extinction dynamics in mainland-island metapopulations: an N-patch stochastic model. Bulletin of Mathematical Biology 64: 913-958.

Amarasekare, P. (1998a). Interactions between local dynamics and dispersal: insights from single species models. Theoretical Population Biology 53: 44-59. 
Amarasekare, P. (1998b). Allee effects in metapopulation dynamics. American Naturalist 152: 298-302.

Amarasekare, P. and H.P. Possingham (2001). Patch dynamics and metapopulation theory: the case of successional species. Journal of Theoretical Biology 209: 333344.

Berec, L., D.S. Boukal and M. Berec (2001). Linking the Allee effect, sexual reproduction, and temperature-dependent sex determination via spatial dynamics. American Naturalist 157: 217-230.

Brassil, C.E. (2001). Mean time to extinction of a metapopulation with an Allee effect. Ecological Modelling 143: 9-16.

Brown, J.H. and A. Kodric-Brown (1977). Turnover rate in insular biogeography: effect of immigration on extinction. Ecology 58: 445-449.

Courchamp, F., T. Clutton-Brock and B. Grenfell (1999). Inverse density dependence and the Allee effect. Trends in Ecology and Evolution 14: 405-410.

Cronin, J.T. and D.R. Strong (1999). Dispersal-dependent oviposition and the aggregation of parasitism. American Naturalist 154: 23-36.

Day, J.R. and H.P. Possingham (1995). A stochastic metapopulation model with variability in patch size and position. Theoretical Population Biology 48: 333-360.

Den Boer, P.J. (1968). Spreading of risk and stabilization of animal numbers. Acta Biotheoretica 18: 165-194.

Etienne, R.S. (2000). Local populations of different sizes, mechanistic rescue effect and patch preference in the Levins metapopulation model. Bulletin of Mathematical Biology 62: 943-958.

Etienne, R.S. (2002). A scrutiny of the Levins metapopulation model. Comments on Theoretical Biology 7: 257-281.

Etienne, R.S. and J.A.P. Heesterbeek (2000). On optimal size and number of reserves for metapopulation persistence. Journal of Theoretical Biology: 203: 33-50.

Etienne, R.S. and J.A.P. Heesterbeek (2001). Rules of thumb for conservation of metapopulations based on a stochastic winking-patch model. American Naturalist 158: 389-407.

Etienne, R.S. and C.J. Nagelkerke (2002). Non-equilibria in small metapopulations: comparing the deterministic Levins model with its stochastic counterpart. Journal of Theoretical Biology 219: 463-478.

Etienne, R.S., M. Lof and L. Hemerik (2002a). The Allee effect in metapopulation dynamics revisited. In: Etienne, R.S. Striking the metapopulation balance. Mathematical models and methods meet metapopulation management. pp. 71-78. $\mathrm{PhD}$ Thesis, Wageningen University, Wageningen, The Netherlands.

Etienne, R.S , B. Wertheim, L. Hemerik, P. Schneider and J.A. Powell (2002b). The interaction between dispersal, the Allee effect and scramble competition affects population dynamics. Ecological Modelling 148: 153-168.

Frank, K. and C. Wissel (1998). Spatial aspects of metapopulation survival - from model results to rules of thumb for landscape management. Landscape Ecology 13: 363-379.

Goel, N.S. and N. Richter-Dyn (1974). Stochastic models in biology. Academic Press, New York, NY.

Gog, J., R. Woodroffe and J. Swinton (2002). Disease in endangered metapopulations: the importance of alternative hosts. Proceedings of the Royal Society of London B 269: 671-676. 
Gotelli, N.J. and W.G. Kelley (1993). A general model of metapopulation dynamics. Oikos 68: 36-44.

Gyllenberg, M. and I. Hanski (1992). Single-species metapopulation dynamics: a structured model. Theoretical Population Biology 42: 35-61.

Gyllenberg, M. and I. Hanski (1997). Habitat deterioration, habitat destruction, and metapopulation persistence in a heterogenous landscape. Theoretical Population Biology 52: 198-215.

Gyllenberg, M. and D.S. Silvestrov (1994). Quasi-stationary distributions of a stochastic meta-population model. Journal of Mathematical Biology 33: 35-70.

Gyllenberg, M., A.V. Osipov and G. Söderbacks (1996). Bifurcation analysis of a metapopulation model with sources and sinks. Journal of Nonlinear Science 6: 329-366.

Gyllenberg, M., I. Hanski and A. Hastings (1997). Structured metapopulation models. In: Hanski, I.A. and M.E. Gilpin (Eds). Metapopulation biology: ecology, genetics, and evolution. Academic Press, San Diego, CA. pp. 93-122.

Gyllenberg, M., J. Hemminki and T. Tammaru. (1999). Allee effects can both conserve and create spatial heterogeneity in population densities. Theoretical Population Biology 56: 231-242.

Hanski, I. (1983). Coexistence of competitors in patchy environment. Ecology 64: 493-500.

Hanski, I. (1994). A practical model of metapopulation dynamics. Journal of Animal Ecology 63: 151-162.

Hanski, I. (1999). Metapopulation ecology. Oxford University Press, Oxford, U.K.

Hanski, I. and M. Gyllenberg (1993). Two general metapopulation models and the core-satellite species hypothesis. American Naturalist 142: 17-41.

Hanski, I. and D.-Y. Zhang (1993). Migration, metapopulation dynamics and fugitive co-existence. Journal of Theoretical Biology 163: 491-504.

Hanski, I. and O. Ovaskainen (2000). The metapopulation capacity of a fragmented landscape. Nature 404: 755-758.

Hanski, I., A. Moilanen and M. Gyllenberg (1996). Minimum viable metapopulation size. American Naturalist 147: 527-541.

Harding, K.C. and J.M. McNamara (2002). A unifying framework for metapopulation dynamics. American Naturalist 160: 173-185.

Hastings, A. (1991). Structured models of metapopulation dynamics. Biological Journal of the Linnean Society 42: 57-70.

Hastings, A. (1995). A metapopulation model with population jumps of varying sizes. Mathematical Biosciences 128: 285-298.

Hess, G.R. (1994). Conservation corridors and contagious disease: a cautionary note. Conservation Biology 8: 256-262.

Hess, G.R. (1996). Disease in metapopulation models: implications for conservation. Ecology 77: 1617-1632.

Hess, G.R. and R.A. Fischer (2001). Communicating clearly about conservation corridors. Landscape and Urban Planning 55: 195-208.

Holt, R.D. (1997). From metapopulation dynamics to community structure: some consequences of spatial heterogeneity. In: Hanski, I.A. and M.E. Gilpin (Eds). Metapopulation biology: ecology, genetics, and evolution. Academic Press, San Diego, CA. pp. 149-164. 
Keitt, T.H., M.A. Lewis and R.D. Holt (2001). Allee effects, invasion pinning, and species' borders. American Naturalist 157: 203-216.

Keymer, J.E., P.A. Marquet, J.X. Velasco-Hernández and S.A. Levin (2000). Extinction thresholds and metapopulation persistence in dynamic landscapes. American Naturalist 156: 478-494.

Lande, R. (1998). Demographic stochasticity and Allee effect on a scale with isotropic noise. Oikos 83: 353-358.

Lande, R., S. Engen and B-E. Saether (1998). Extinction times in finite metapopulation models with stochastic local dynamics. Oikos 83: 383-389.

Levins, R. (1969). Some demographic and genetic consequences of environmental heterogeneity for biological control. Bulletin of the Entomological Society of America 15: 237-240.

Levins, R. (1970). Extinction. In: Gertenhaber, M. (Ed.). Some mathematical problems in biology. American Mathematical Society, Providence, RI. pp. 75-107.

Levins, R. and D. Culver (1971). Regional coexistence of species and competition between rare species. Proceedings of the National Academy of Science of the USA 68: $1246-1248$.

McCarthy, M.A. (1997). The Allee effect, finding mates and theoretical models. Ecological Modelling 103: 99-102.

Nagelkerke, C.J. and S.B.J. Menken (2002). Local vs. global power. Coexistence of specialist and generalist metapopulations. Manuscript.

Nee, S. and R.M. May. (1992). Dynamics of metapopulations: habitat destruction and competitive coexistence. Journal of Animal Ecology 61: 37-40.

Nee, S., R.M. May and M.P. Hassell (1997). Two-species metapopulation models. In: Hanski, I.A. and M.E. Gilpin (Eds). Metapopulation biology: ecology, genetics, and evolution. Academic Press, San Diego, CA. pp. 123-147.

Ovaskainen, O. (2001). The quasi-stationary distribution of the stochastic logistic model. Journal of Applied Probability 38: 898-907.

Ovaskainen, O. and I. Hanski (2001). Spatially structured metapopulation models: global and local assessment of metapopulation capacity. Theoretical Population Biology 60: 281-302.

Ovaskainen, O., K. Sato, J. Bascompte and I. Hanski (2002). Metapopulation models for extinction threshold in spatially correlated landscapes. Journal of Theoretical Biology 215: 95-108.

Ray, C., M. Gilpin and A.T. Smith. (1991). The effect of conspecific attraction on metapopulation dynamics. Biological Journal of the Linnean Society 42: 123-134.

Reed, J.M. (1999). The role of behavior in recent avian extinctions and endangerments. Conservation Biology 13: 232-241.

Sabelis, M., O. Diekmann and V.A.A. Jansen (1991). Metapopulation persistence despite local extinction: predator-prey patch models of the Lotka-Volterra type. Biological Journal of the Linnean Society 42: 267-283.

Slatkin, M. (1974). Competition and regional coexistence. Ecology 55: 128-134.

Stephens, P.A. and W.J. Sutherland (1999). Consequences of the Allee effect for behaviour, ecology and conservation. Trends in Ecology and Evolution 14: 401405 .

Stephens, P.A., W.J. Sutherland and R.P. Freckleton (1999). What is the Allee effect? Oikos 87: 185-190. 
Taneyhill, D.E. (2000). Metapopulation dynamics of multiple species: the geometry of competition in a fragmented habitat. Ecological Monographs 70: 495-516.

Vandermeer, J. and R. Carvajal (2001). Metapopulation dynamics and the quality of the matrix. American Naturalist 158: 211-220.

Wissel, C. (1994). Stochastic extinction models discrete in time. Ecological Modelling 75: 183-192.

Rampal S. Etienne

Community and Conservation Ecology Group, University of Groningen

Lia Hemerik

Biometris, Wageningen University and Research Centre 